Research Paper

\title{
The Viral Oncoprotein HBx of Hepatitis B virus Promotes the Growth of Hepatocellular Carcinoma through Cooperating with the Cellular Oncoprotein RMP
}

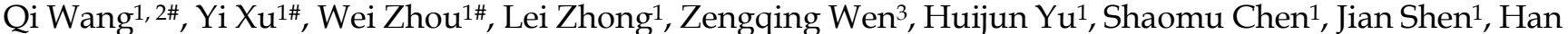 \\ Chen ${ }^{1}$, Qinying She ${ }^{1}$, Jingting Jiang ${ }^{2}$, Jingcheng Miao ${ }^{1,}{ }^{\otimes}$ and Wenxiang Wei ${ }^{1,}$
}

1. Department of Cell Biology, School of Medicine, Soochow University, Suzhou, 215123 China

2. Department of Tumor Biotherapy, Third Affiliated Hospital of Soochow University, Changzhou, 213003 China

3. Eastern Hepatobiliary Surgery Hospital, Shanghai, 200433, China

\# These authors contribute equally.

$\triangle$ Corresponding authors: Wenxiang Wei or Jingcheng Miao, Department of Cell Biology, School of Medicine, Soochow University, Suzhou, 215123 China; Tel: 86-512-5188-0107. Email: wenxiangw@suda.edu.cn, or mjc@suda.edu.cn

(c) Ivyspring International Publisher. This is an open-access article distributed under the terms of the Creative Commons License (http://creativecommons.org/ licenses/by-nc-nd/3.0/). Reproduction is permitted for personal, noncommercial use, provided that the article is in whole, unmodified, and properly cited.

Received: 2014.08.05; Accepted: 2014.10.14; Published: 2014.11.18

\begin{abstract}
The smallest gene HBx of Hepatitis B virus (HBV) is recognized as an important viral oncogene (V-oncogene) in the hepatocarcinogenesis. Our previous work demonstrated that RMP is a cellular oncogene (C-oncogene) required for the proliferation of hepatocellular carcinoma ( $\mathrm{HCC})$ cells. Here we presented the collaboration between V-oncogene HBx and C-oncogene RMP in the development of HCC. The coexpression of HBx and RMP resulted in the cooperative effect of antiapoptosis and proliferation of HCC cells. In vivo, overexpression of RMP accelerated the growth of $\mathrm{HBx}$-induced xenograft tumors in nude mice and vice versa $\mathrm{HBx}$ promoted the growth of RMP-driven xenograft tumors. Although HBx didn't regulate the expression of RMP, HBx and RMP interact with each other and collocalized in the cytoplasm of $\mathrm{HCC}$ cells. $\mathrm{HBx}$ and RMP collaboratively inhibited the expression of apoptotic factors and promoted the expression of antiapoptotic factors. This finding suggests that HBV may induce, or at least partially contributes to the carcinogenesis of $\mathrm{HCC}$, through its $\mathrm{V}$-oncoprotein $\mathrm{HBx}$ interacting with the $\mathrm{C}$-oncoprotein RMP.
\end{abstract}

Key words: HBx, viral oncogene

\section{Introduction}

A leading cause for the development of hepatocellular carcinoma (HCC) is chronic infection with Hepatitis B Virus (HBV). More than half of HCC cases worldwide are due to chronic HBV infection. HBV infection-related liver diseases remain a major global health problem (1).

Within the 3.2 kilobases $(\mathrm{Kb}) \mathrm{HBV}$ genome, there are four overlapping genes encoding the viral core protein (capsid), surface proteins (envelope), reverse transcriptase, and $X$ protein $(\mathrm{HBx})$.
$\mathrm{HBx}$ is a major regulator encoded by HBV genome, which plays an important role in the development of HBV-associated HCC. Chronic HBV infection develops into HCC through several factors, including the integration of viral DNA into the genome of the host cell, the chronic inflammation due to the immune response of the host to the HBV infection. As a typical viral oncogene (V-oncogene) of $\mathrm{HBV}, \mathrm{HBx}$ is predominantly nuclear at low expression levels and mostly cytoplasmic at high expression levels. Some of 
cytosolic HBx are localized in the outer mitochondrial membrane (2-4).

RNAs are synthesized by RNA polymerases (RNAPs). In eukaryotic cells, different kinds of RNAs are transcribed by RNAP I, II and III in the nuclear genome (5). RNAP I, II and III are composed of 14, 12 and 17 subunits respectively, among which RPB5, RPB6 and RPB8 are commonly shared by all three RNAPs (6). HBx has been identified to associate with RPB5 subunit of RNAP II and regulate transcription activity (7). Further, from HepG2 cell line, we identified RPB5-mediating protein (RMP) with RPB5 as a probe (8). We found that the general transcriptional factor TFIIF was associated with RNAP II through the interaction between RAP30 and RPB5 subunits. In yeast, the biosynthesis and assembly of eukaryotic RNA polymerase required both RMP and RPB5. Deletion of RMP led to abnormally substantial accumulation of all three RNAPs of I, II and III in the cytoplasm (5).

There are multiple variants of RMP, including the unconventional prefolding RPB5 interactor (URI). Recently, both Dr. Krek group and ours showed that RMP exhibited the typical activity of a cellular oncogene (C-oncogene) during the carcinogenesis $(9,10)$. URI, which was overexpressed in tissues and cell lines of human ovarian carcinomas, was required for the survival of ovarian cancer cells (9). The molecular mechanism analysis revealed that URI associated and inactivated PP1Y (phosphatase 1 gamma), which enhanced the S6K1 survival signaling (11). The release of PP1 $\gamma$ by the phosphorylation of URI results in apoptosis and cell death. The research of our group found that RMP is actively involved in the proliferation of HCC cells (10). RMP presents an antiapoptotic role, which in turn supports the proliferation and growth of HCC cells. Depletion of RMP induced apoptosis of HCC cells. RMP is also required for development and growth of HCC xenograft tumors in vivo. Interestingly, RMP has been shown to play distinct roles in normal hepatic cells and HCC cells (12).

Despite of many studies conducted toward HBV and $\mathrm{HBx}$, the mechanism of how HBx induces the carcinogenesis of HCC remains largely obscure. As both HBx and RMP associate with RNAP by interacting with RPB5 and demonstrate the oncogenic property, we investigated the relationship between HBx and RMP, the two oncogenes in the carcinogenesis of HCC.

In this article, we identified the collaboration between HBx and RMP. The two oncogenes cooperatively stimulated the proliferation of HCC cells, demonstrating an antiapoptotic effect on the HCC cells, which contributed to hepaticcarcinogenesis.

\section{Materials and Methods}

\section{Plasmids, Cell Lines and Reagents.}

The plasmid pFlagCMV4 was from Sigma. pGPU6-Neo was from GenePharma Co. (Shanghai, China). pCDNA3.1/Neo/RMP and pEx-5/GFP/ $\mathrm{Neo} / \mathrm{HBx}$ were constructed by and purchased from GenePharma Co. (Shanghai, China). The plasmids pGPU6/Neo-SCR and pGPU6/Neo-RMPi for RMP depletion were constructed as described previously (10). HepG2 cell line stably expressing HBx $(13,14)$ was a gift from Dr. Xiaodong Zhang in Nankai University. All other cell lines used in this article were obtained from our own laboratory of the Department of Cell Biology at Soochow University. Cells were cultured in DMEM medium (Gibco-BRL, Shanghai, China) supplemented with $10 \%$ Fetal Bovine Serum (FBS) (Sino-American Biotechnology Co, Shanghai, China). The Annexin-V-FITC/PI apoptosis detection kit was from BD Biosciences (Shanghai, China). Methyl-thiazolyl-tetrazolium (MTT) was from Sigma (Shanghai, China). Cell Counting Kit-8 (CCK-8) was purchased from Dojindo Laboratories (Shanghai, China).

Antibodies against Bax, Bad, Bcl-2, p53, AFP and $\beta$-actin were purchased from Boster Company (Wuhan, China). The RMP antibody was a product from AppTec Pharmaceutical (Suzhou, China). Antibody against HBx was purchased from Santa Cruz Biotechnology, Inc (Shanghai, China).

\section{Cell Culture, Transfection, Western Blot Analysis and Immunoprecipitation.}

A total of $2 \times 10^{5}$ cells were seeded into each well of a 24-well plate, $24 \mathrm{hrs}$ prior to transfection. Cell numbers were chosen to finally reach $90 \%$ confluency at the time of transfection. These cells were transfected with lipofectamine 2000 (Invitrogen, Shanghai, China) according to the manufacturer's protocol. LF2000/DNA complexes were allowed to form in OptiMEM with final concentration of $10 \mu \mathrm{g} / \mathrm{ml}$ LF2000. Selection of stably transfected cells were conducted as previously described $(10,15)$.

For immunoprecipitation, the supernatants of cell lysates were collected after centrifugation and immunoprecipitated with $20 \mu \mathrm{l}$ of $50 \%$ anti-FLAG M2 or $\mathrm{HBx}$ resin, followed by rotation for $2 \mathrm{hrs}$ at $4^{\circ} \mathrm{C}$. Then the resin was washed 4 times with buffer (50 $\mathrm{mM}$ Tris- $\mathrm{HCl}, \mathrm{pH} 7.5,150 \mathrm{mM} \mathrm{NaCl}$ ) (TBS). The bound proteins were eluted, fractionated by $12.5 \%$ SDS-PAGE, transferred onto PVDF membranes, and subjected to Western blot analysis with the antibody. The proteins were visualized by enhanced chemiluminescence (ECL), according to the manufacturer's instructions (Amersham Pharmacia Biotech) $(10,15)$. 


\section{Cell Viability Assay.}

Cell proliferation assay by Cell Counting Kit-8 (CCK-8) was conducted as following. Cells were seeded into a 96 -well plate $\left(5 \times 10^{4}\right.$ cells/cell $)$ and cultured in $100 \mu \mathrm{l}$ of DMEM supplemented with $10 \%$ FBS. At the indicated time points, medium was exchanged for $110 \mu \mathrm{l}$ of DMEM with CCK-8 reagent (10 $\mu \mathrm{l} \mathrm{CCK-8}$ and $100 \mu \mathrm{l}$ DMEM), and the cells were incubated for 2 hrs. Absorbance was measured at a wavelength of $450 \mathrm{~nm}$ for each well, with the reference wavelength set at $600 \mathrm{~nm}$. An increase or decrease in absorbance values at $450 \mathrm{~nm}$ in the experimental wells relative to the initial value indicated cell growth or death, respectively. Cell growth was monitored every 24 hrs over 5 days. All experiments were independently repeated at least three times.

MTT assays were carried out as described previously $(10,15)$. Briefly, cells were cultured 96-well plate for $24 \mathrm{hrs}$, and $30 \mu \mathrm{l}$ of MTT $(5 \mathrm{mg} / \mathrm{ml})$ was added to each well. Crystals were formed and then dissolved by adding $300 \mu \mathrm{l}$ isopropanol acidified with $\mathrm{HCl}(0.04 \mathrm{~N})$ containing $10 \%$ Triton X-100. Finally, $200 \mu \mathrm{l}$ of the blue formazan mixtures was transferred to 96-well plates. Microplate Reader (Model 550, BIO-RAD, Shanghai, China) was used to read the plates at $570 \mathrm{~nm}$.

\section{Analysis of Apoptosis and cell cycle by Flow Cytometry.}

Cells were exposed to ionising radiation (IR) using a ${ }^{60} \mathrm{Co} \gamma$-irradiator at a dose of 2 or $6 \mathrm{~Gy}$. Cells radiated or unradiated were then stained with Annexin V-FITC and propidium iodide (PI) and analyzed for apoptosis according to the manufacturer's instructions (BD Biosciences, Shanghai, China). Briefly, cells were washed with buffer for 2 times, and re-suspended in $400 \mu \mathrm{l}$ of Dulbecco's phosphate-buffered saline (PBS). Then, $100 \mu \mathrm{l}$ of cell suspension was incubated with $10 \mu \mathrm{l}$ of PI $(50 \mu \mathrm{g} / \mathrm{ml})$ and $5 \mu$ l Annexin V-FITC for 15 minutes at room temperature (RT) in the dark, followed by the analysis with flow cytometry. Cells that stained positive for only Annexin V-FITC were in the early stage of apoptosis, whereas cells that stained positive for both Annexin V-FITC and PI were in the stage of late apoptosis or primary necrosis.

\section{Tumor Formation Assay in Nude Mice.}

Tumor formation assay was carried out as described previously $(10,12)$. Xenograft tumors developed in the nude mice after inoculation were subjected to treatment by vectors of RMP overexpression, RMP depletion or HBx. Briefly, a suspension ( $25 \mu \mathrm{l} /$ mouse) of Lipofectamine $2000(20 \mu \mathrm{g})$ was mixed with expression plasmids, empty vector $(10 \mu \mathrm{g})$ or PBS in a final concentration of $100 \mu \mathrm{g} / \mathrm{ml}$ and incubated for $20 \mathrm{~min}$ to allow them to complex. Then the complex was delivered by multiple intratumor injection every other day for 2 weeks. At the end of the fourth week, tumors were dissociated and evaluated. The animal operations and procedures were approved by the Committee on the Use of Live Animals in Teaching and Research of Soochow University.

\section{Reverse transcription and Quantitative real time Polymerase Chain Reaction (qRT-PCR).}

Total RNA was extracted using the RNeasy Mini Kit (Qiagen, Shanghai, China). Thermoscript RT system (Invitrogen, Shanghai, China) was applied for the reaction of reverse-transcription. The real time PCR was performed in triplicate in a total volume of $20 \mu 1$ containing $10 \mu \mathrm{l}$ SsoFastEvaGreensupermix (Bio-Rad) with SYBR Green, $2 \mu \mathrm{l}$ cDNA, $2 \mu \mathrm{l}$ each of the primers, and $6 \mu \mathrm{l}$ RNase-free water. The PCR program was $94^{\circ} \mathrm{C}$ for $2 \mathrm{~min}$, followed by 40 cycles at $94^{\circ} \mathrm{C}$ for $15 \mathrm{~s}$, $60^{\circ} \mathrm{C}$ for $15 \mathrm{~s}$, and $72^{\circ} \mathrm{C}$ for $30 \mathrm{~s}$. qRT-PCR assay was run on a Mini OpticonTM Real-time PCR instrument. Each reaction contains 3 technical replicates for qRT-PCR analysis. Primers for RMP were $5^{\prime}$ - TCC GAA TAA ATA CTG GAA AG $-3^{\prime}$ and $5^{\prime}$-AAG GCT CTG TAA ATG TCT GC -3'. Primers for Bax were 5'TTT TGC TTC AGG GTT TCA TC $-3^{\prime}$ and $5^{\prime}-$ GAC ACT CGC TCA GCT TCT TG -3'. Primers for Bcl-2 were 5' - GGT GGG AGG GAG GAA GAA - $3^{\prime}$ and 5'CGC AGA GGC ATC ACA TCG -3'. Primers for GAPDH were 5'-GAC CTG ACC TGC CGT CTA-3' and $5^{\prime}$ - AGG AGT GGG TGT CGC TGT $-3^{\prime}$. Primers for HBx were $5^{\prime}$-ACCGACCTTGAGGCCTACTT-3' and 5'-GCTTGGCAGAGGTGAAAAAG-3'.

\section{Immunohistochemistry Detection.}

Tissues of nude mice were subjected to formalin fixation, paraffin embedding and sectioning for immunohistochemistry assays as described previously (16). The slides were first blocked in PBST containing $3 \%$ BSA (PBST-BSA) at $37^{\circ} \mathrm{C}$ for 30 minutes and incubated overnight at $4^{\circ} \mathrm{C}$ with mouse monoclonal antibodies (1:500 in PBST-BSA). Slides were then washed 3 times in PBST (10 minutes each time) and incubated for 2 hrs with the secondary antibody (anti-mouse HRP, 1:200) in PBST at room temperature. Slides were then washed 3 times in PBST and mounted with $3 \mu \mathrm{l}$ of Vectashield for further analysis. Finally sections were examined at high power ( $x 400)$ under a standard light microscope. Cell staining was regarded as positive if nuclear was homogeneous stained or $10 \%$ or more of cytoplasm was heterogeneously stained. 


\section{Immunofluorescence and confocal microsco-}

py.

HepG2 cells were grown on glass coverslips, washed once in PBS, and fixed with $2 \%$ formaldehyde in PBS for $30 \mathrm{~min}$ at room temperature (RT). Cells were then permeabilized for 5 min with $100 \%$ cold methanol, and dried at $-25^{\circ} \mathrm{C}$. GFP-fused proteins were detected after being counterstained with 0.0005\% Evans Blue. For immunostaining, coverslips were blocked in PBS with 1.5\% BSA at RT for $1 \mathrm{hr}$ and incubated with primary antibodies in a humidifying chamber at RT for $1 \mathrm{hr}$ (1:300 monoclonal antibody). The cells were washed 5 times with PBS and incubated at RT for $1 \mathrm{hr}$ with secondary antibodies (1:30 Cy3-labled goat anti-rabbit IgG and FITC-labled goat anti-mouse IgG). Subsequently, the cells were washed 5 times in PBS and mounted with Vectashield mounting medium. A confocal laser scanning microscope (TCS-SP2, Leica Co., Ltd) was applied to acquire the immunofluorescent images.

\section{Statistical analysis.}

Values are expressed as the mean +/- standard error (SE). Student's t-test was used to determine the significance of the difference between compared groups. $P<0.05$ indicated significant differences.

\section{Results}

\section{HBx and RMP cooperatively promoted the proliferation of HCC cells}

As both HBx and RMP associate with RPB5 of RNA polymerase II and exhibit oncogenic property in the carcinogenesis of HCC, we examined the combined effect of HBx and RMP on the proliferation of HCC cells. HepG2 cells were transfected with expression vectors of $\mathrm{HBx}$ or RMP alone, or both $(\mathrm{RMP}+\mathrm{HBx})$. The expression of RMP and HBx were determined by Western blot analysis. As shown in Fig. 1A, the protein level of RMP was elevated in cells transfected with RMP vector, while HBx expressed only in the cells transfected with HBx vector (lane 4). The expression of both HBx and RMP was enhanced in the cells transfected with both vectors of $\mathrm{HBx}$ and RMP (RMP+HBx, lane 5) (Fig. 1A). Then we examined the effect of RMP and HBx on the growth of HCC cells. Cell Counting Kit- 8 (CCK-8) was used as described in the Material and Methods. As shown in the Fig. 1B, the cells expressing either HBx or RMP grew faster than the control cells untransfected or transfected with vector alone. Interestingly the coexpression of both RMP and HBx resulted in stronger promotion of cell proliferation than either RMP or HBx expression alone, indicating a cooperative effect between RMP and HBx on the growth of HCC cells. To confirm the results, we examined the cells with an alternative method of MTT which was extensively applied to test proliferation $(10,15)$. The results also showed that RMP and HBx enhanced the growth of HCC cells with a cooperative effect (Fig. 1C), which is consistent with the results by CCK-8 (Fig. 1B). We further examined if RMP and HBx cooperated to promote the cell growth in a dose dependent manner. HepG2 cells stably expressing HBx $(13,14)$ was co-transfected with increasing amounts of RMP expression vector. As shown in Fig. 1D, increasing amount of RMP exhibited stronger cooperative promotion of the growth of HCC cells expressing HBx, indicating an interaction and cooperation between HBx and RMP.

\section{The cooperative antiapoptotic effect of $\mathrm{HBx}$ and RMP on HCC cells}

As apoptosis is an important factor affecting cell proliferation, we examined the co-effect of $\mathrm{HBx}$ and RMP on the apoptosis of HCC cells. Cells transfected with various vectors were subjected to apoptosis analysis by flow cytometry. As shown in Fig. 2A, there was decreased population of both early and late apoptotic cells in HepG2 overexpressing either HBx or RMP, compared with cells untransfected or transfected with empty vector. Apparently, HepG2 cells co-expressing both HBx and RMP demonstrated the least apoptotic cells among all five groups of transfected and untransfected cells (Fig. 2A and 2C, left panel). To confirm the results, the effect of $\mathrm{HBx}$ and RMP on the induced apoptosis was also carried out by using ${ }^{60} \mathrm{Co}$ irradiation (Fig. 2B). More than half population of untreated HCC cells (Unt) or cells treated with empty vector (vector) were in early and late apoptotic phase after treatment of radiation. In contrast, the late apoptotic cells were significantly decreased in the HCC cells expressing either RMP or HBx. The least late apoptotic cells were observed in the HCC cells expressing both RMP and HBx, although early apoptosis remained constant in all the transfected or untreated cells (Fig. 2B and 2C, right panel). These results suggest that RMP and HBx exert a cooperatively antiapoptotic effect on the HCC cells.

\section{RMP promoted the growth of HCC xenograft tumors induced by $\mathbf{H B x}$}

We also examined the effect of RMP on the xenograft tumors developed from $\mathrm{HBx}$-expressing HepG2 cells. Mice were inoculated with HepG2 cells which stably expressed HBx as detected by Western blot (Fig.1A). Tumors were developed to considerable size 2 weeks later. Then the mice were treated by intratumor injection with RMP expression plasmid (RMPo), RMP depletion plasmid (RMPi), empty vec- 
tor or PBS, respectively. As shown in Fig. 3A, the overexpression of RMP accelerated HBx-induced growth of HCC xenograft tumors (HBx+RMPo), while the depletion of RMP retarded the growth of xenograft tumors ( $\mathrm{HBx}+\mathrm{RMPi})$, compared with the control tumors treated with PBS or empty vector. As results, RMPo enlarged the HCC xenogroft tumors, while RMPi dramatically reduced the size of the tumors (Fig. 3B and 3C), suggesting a collaboration between $\mathrm{HBx}$ and RMP in promoting the growth of HCC tumors.

\section{HBx promoted the growth of HCC xenograft tumors induced by RMP}

To further confirm the cooperation of RMP and $\mathrm{HBx}$ on the growth of HCC tumor, we examined the effect of HBx on the growth of HCC xenograft driven by RMP overexpression. Previously we established xenograft tumors with SMMC-7721 cells stably overexpressing RMP (RMPo) which were verified by Western blot $(10,12)$. Two weeks after inoculation with RMP-expressing 7721 cells, the developed tumors were administrated with $\mathrm{HBx}$ vector, empty vector or PBS, respectively, as indicated. As shown in Fig. 4A, the xenograft tumors grew much faster after the treatment with $\mathrm{HBx}$ vector (RMP+HBx), com-
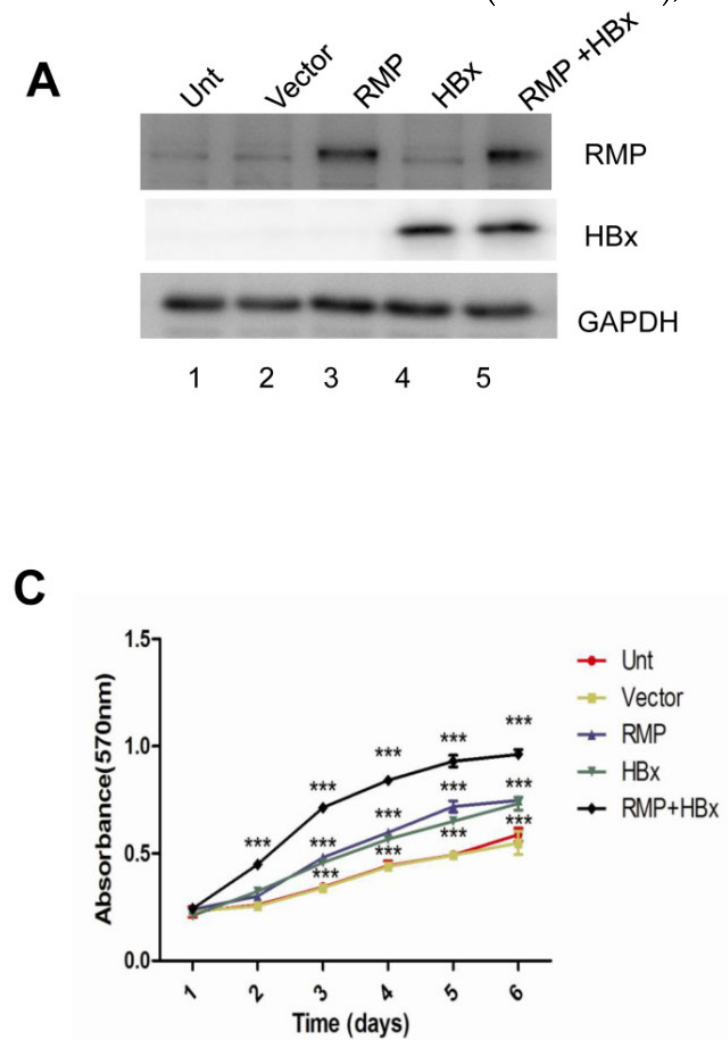

B

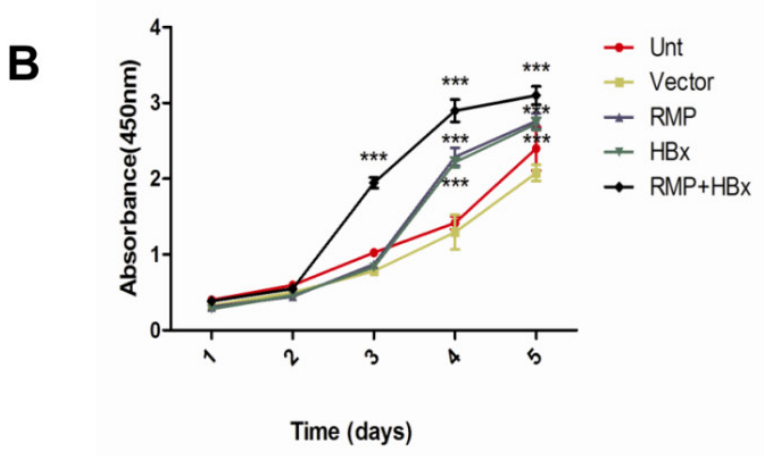

pared with the control tumors treated with PBS or empty vector (Vector). As results, the expression of HBx promoted the RMP-induced tumors, resulting in the biggest tumors among all 3 groups (Fig. 4B and $4 C)$.

\section{HBx did not regulate the expression of RMP in HCC cells}

As $\mathrm{HBx}$ has been shown to cooperate with oncogenes by upregulating their expression $(13,14,17)$, we examined if $\mathrm{HBx}$ regulated the expression of endogenous oncogene RMP in HCC cells. As shown in Fig. 5A, the transcription of RMP mRNA was not affected by transfection with increasing amount of $\mathrm{HBx}$ expression vectors, as detected by qRT-PCR. Then we further examined whether HBx regulated translation, stability or degradation of RMP protein. As shown in Fig.5B, the expression of RMP protein was neither affected by the transfection with increasing amount of $\mathrm{HBx}$ expression vectors, demonstrating that HBx did not regulate the expression of RMP at either transcription or translation levels. These results suggest that the cooperation between HBx and RMP is not mediated by the regulation of RMP expression, but by the possible interaction between HBx and RMP.

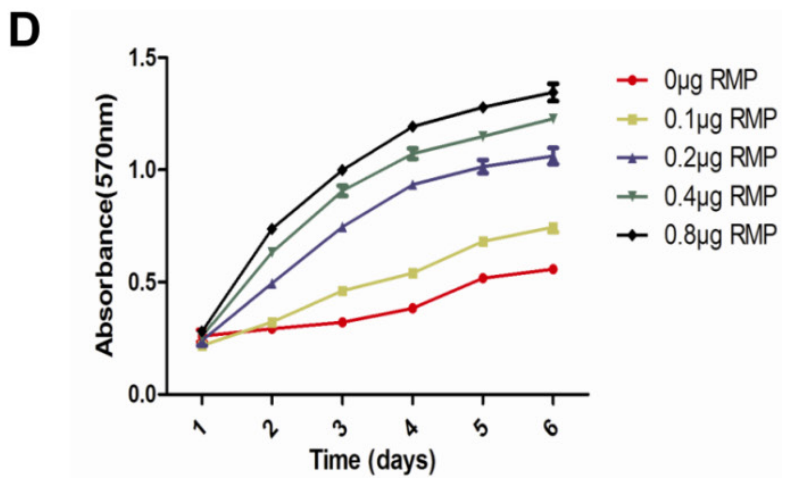

Fig. 1. HBx and RMP cooperatively promote the proliferation of HCC cells. (A). HepG2 cells were transfected with vectors of RMP, HBx or both vectors of RMP and HBx. The untransfected (Unt) cells or cells transfected with empty vector (Vector) were applied as controls. Cell lysates were extracted for the Western blot analysis with the antibodies against RMP, HBx or GAPDH. (B) and (C). HBx and RMP cooperatively promote the proliferation of HCC cells. HepG2 cells were transfected with vectors as indicated. CCK8 (B) and MTT (C) assays were carried out on the days indicated for the determination of cell growth as described in Materials and Methods. (D). Dose-dependent effect of $\mathrm{HBx}$ and RMP on the growth of HCC cells. HepG2 cells stably expressing HBx were transiently transfected with increasing amount of RMP expression vectors as indicated. MTT assay was carried out on the days indicated for the determination of cell growth. Each measurement was made in triplicate. Student's t-test, $* * *, p<0.001$, relative to controls. 

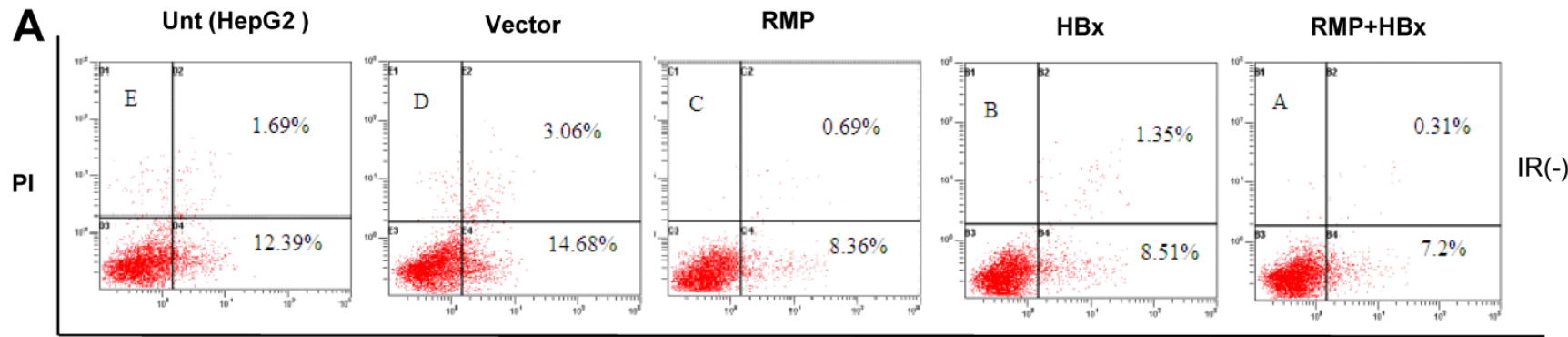

Annexin-V
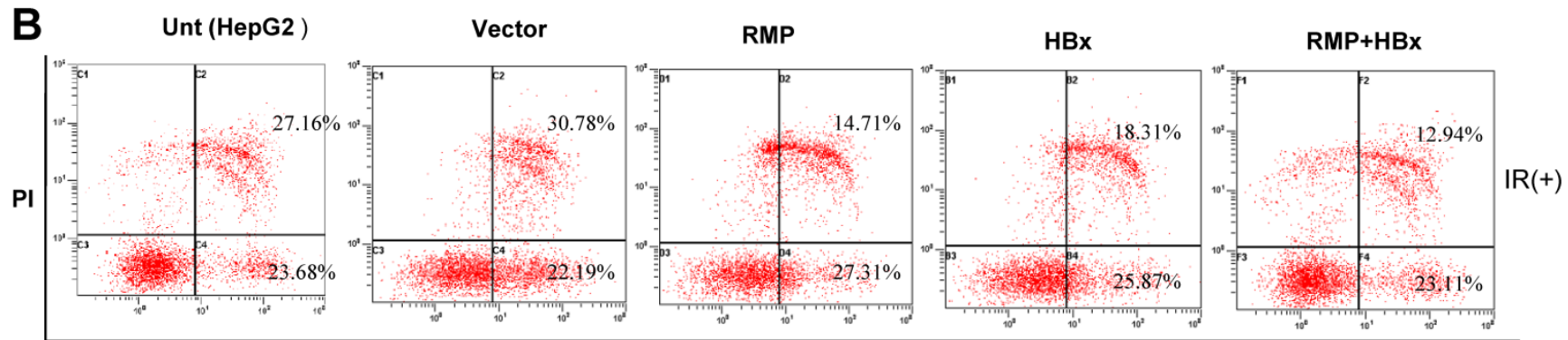

Annexin-V
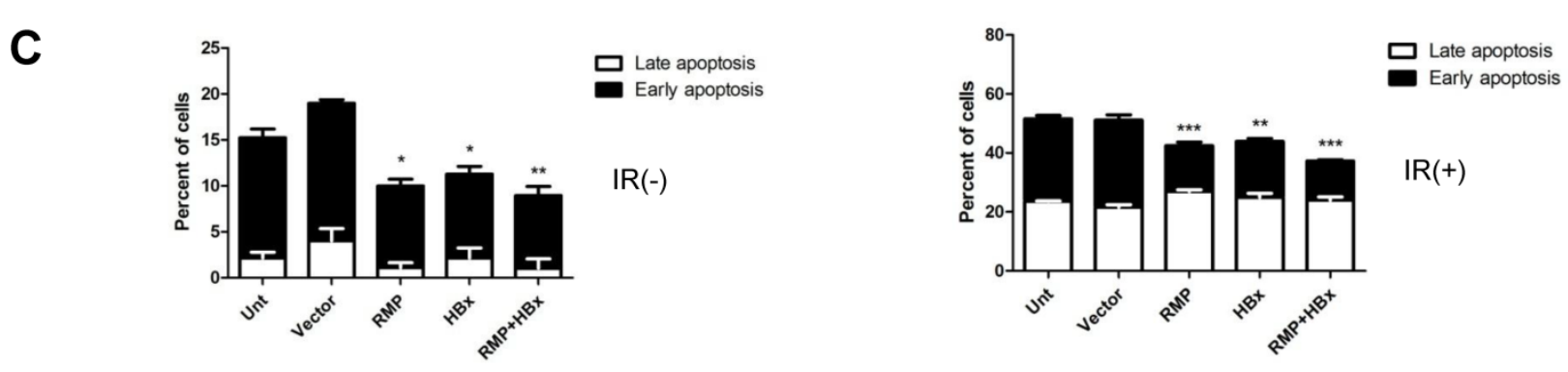

Fig. 2. The cooperative effect of HBx and RMP in the antiapoptosis of HCC cells. (A). HepG2 cells were transfected with expression vectors as indicated. Two days later, cells were harvested and subjected to apoptosis analysis by flow cytometry. The percentage of apoptotic cells were scored and depicted graphically in (C, left panel). (B). HepG2 cells were transfected with expression vectors as indicated. One day later, cells were irradiated at $6 \mathrm{~Gy}$ and cultured for additional 2 days. Finally cells were harvested and subjected to apoptosis analysis by flow cytometry. The percentage of apoptotic cells were scored and depicted graphically in (C, right panel). Student's t test, *, $p<0.05$; **, $p<0.01 ; * * *, p<0.001$, relative to controls.

\section{HBx interacted with RMP in HCC cells}

As no evidence showed that $\mathrm{HBx}$ could regulate the expression of RMP, we wondered if there was an interaction between HBx and RMP. At first, we examined the localization and colocalization of HBx and RMP in HCC cells. The immunofluorescent staining was performed with HepG2 cells transfected with both RMP and HBx vectors. As shown in Fig. 6A, both RMP and HBx were localized primarily in the cytoplasm and less in the nucleus of HCC cells. The merged image demonstrated that RMP and HBx colocalized in the cytoplasm of HepG2 cells.

Then immuoprecipitation was also performed to determine the molecular interaction between $\mathrm{HBx}$ and RMP. The results were shown in Fig. 6B, HCC cells were cotransfected with vectors encoding $\mathrm{HBx}$ and Flag-RMP. The exogenously expressed Flag-RMP was immunoprecipitated by Flag antibody. The Western blot analysis showed that HBx was coimmunoprecip- itaed with Flag-RMP in contrast to the control immunoprecipitation by IgG (Fig. 6B).

We further examined if the HBx could interact with the endogenous RMP. The lysates of HepG2 cells stably transfected with $\mathrm{HBx}$ was incubated with an antibody against HBx. The immunoprecipitates were examined by Western blot analysis with both HBx and RMP antibodies. As shown in Fig. 6C, the endogenous RMP was immunoprecipitated together with $\mathrm{HBx}$, indicating that $\mathrm{HBx}$ was also associated with endogenous RMP which may be within a complex of RNA polymerase II.

\section{HBx and RMP collaboratively regulated the apoptotic genes}

To investigate the mechanism of the collaboration between HBx and RMP on HCC growth, we examined the effect of HBx or RMP on the expression of apoptotic genes. As shown in Fig. 7A, both RMP and $\mathrm{HBx}$ suppressed the expression of proapoptotic factor Bax. The co-expression of RMP and HBx 
demonstrated even stronger inhibition on Bax expression than either HBx or RMP alone (Fig. 7A and 7B). In contrast, the expression of antiapoptotic factor Bcl-2 was increased by RMP or HBx. Co-transfection of RMP and $\mathrm{HBx}$ expression vectors resulted in stronger expression of Bcl-2 than either HBx or RMP alone (Fig.7A and 7B).

The collaborative effect of RMP and HBx was also observed on the expression of p53 and caspase-3, which was detected by qRT-PCR (Fig. 7C). The expression of p53 was inhibited by either RMP or HBx to some extents. And the cotransfection of HBx and RMP vectors significantly suppressed p53 expression (Fig. 7C).

To confirm the effect of RMP and HBx on the expression of apoptotic genes, we further examined the expression of apoptotic genes in the HCC xeno- graft tumors induced by RMP or HBx. As shown in Fig. 8, RMP expression increased when the xenograft tumors were treated with RMP expression vector (RMPo) and decreased when tumors were treated with RMP interference vector (RMPi). HBx was expressed in the xenograft tumors derived from HepG2 cells which stably expressed the HBx. The expression of AFP was increased by expression of both HBx and RMP. The expression of proapoptotic factor Bax was also decreased by HBx or the coexpression of HBx and RMP (HBx+RMPo), while increased by the depletion of RMP (HBx+RMPi) (Fig. 8). In contrast, the expression of antiapoptotic factor Bcl-2 was increased by $\mathrm{HBx}$ alone or coexpression of $\mathrm{HBx}$ and RMP $(\mathrm{HBx}+\mathrm{RMPo})$, but decreased by the depletion of RMP $(\mathrm{HBx}+\mathrm{RMPi})$.

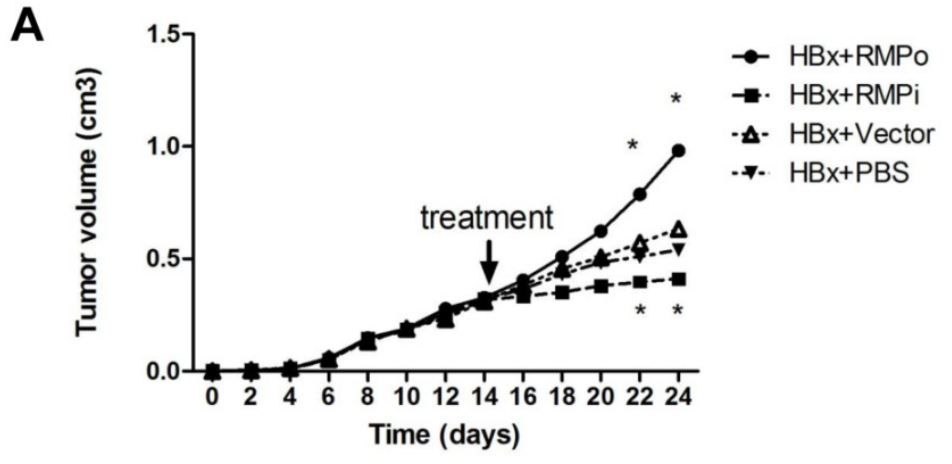

B

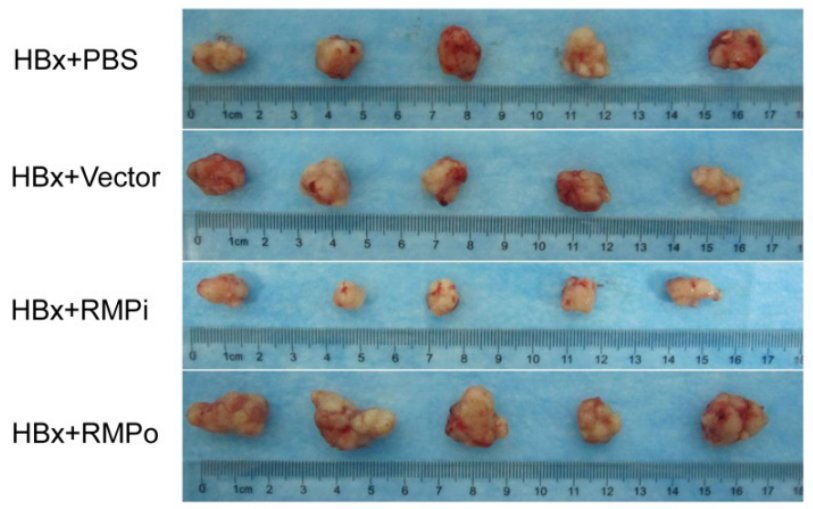

C

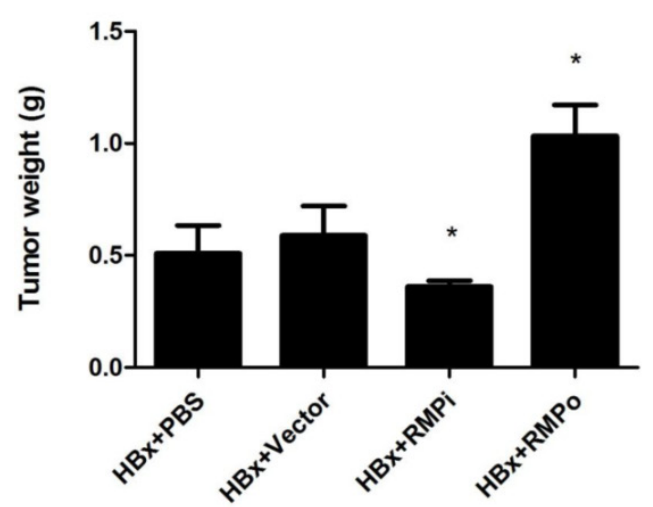

Fig. 3. RMP promotes the growth of HCC xenograft tumors induced by HBx. (A). Nude mice were inoculated subcutaneously with HepG2 cells stably transfected with $\mathrm{HBx}$ expression vector for tumor formation. Two weeks after inoculation, tumors in the nude mice were injected every other day with indicated vectors, or PBS as a control. Tumor size was measured everyday after inoculation and expressed as the mean volume of tumors from five mice, which was depicted graphically. (B). HCC xenograft

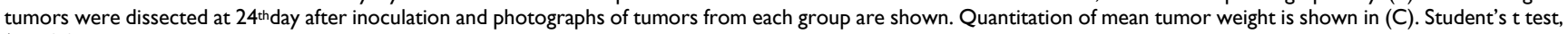
$*, p<0.05$, relative to controls. 
A

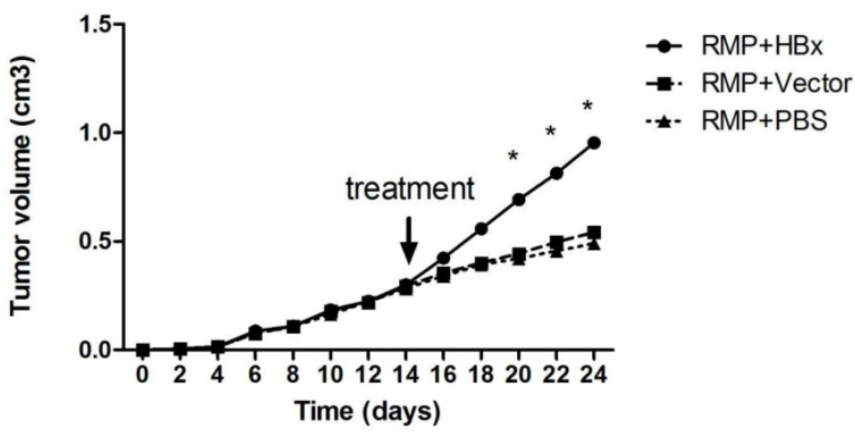

B

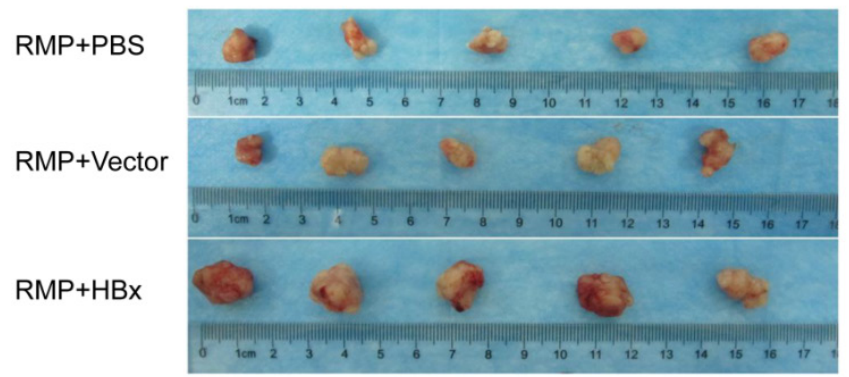

C

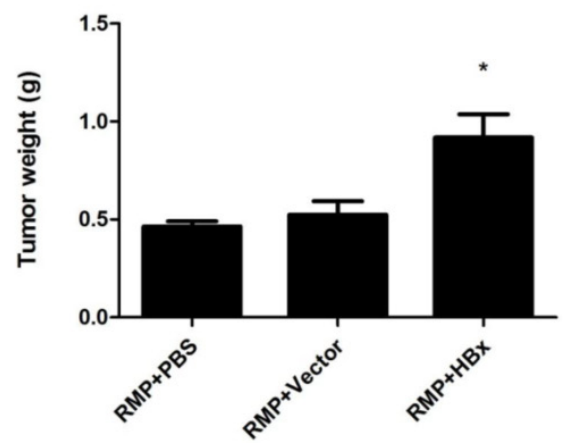

Fig. 4. HBx promotes the growth of HCC xenograft tumors induced by RMP. (A). Nude mice were inoculated subcutaneously with SMMC-772 1 cells stably transfected with RMP expression vectors for tumor formation. Two weeks after inoculation, tumors in the nude mice were injected every other day with indicated vectors, or PBS as a control. Tumor size was measured everyday after inoculation and expressed as the mean volume of tumors from five mice, which was depicted graphically. (B). Tumors were dissected at $24^{\text {th }}$ day after inoculation and photographs of tumors from each group are shown. Quantitation of mean tumor weight is shown in (C). Student's $t$ test, ${ }^{*}, p<0.05$, relative to controls.

\section{A}

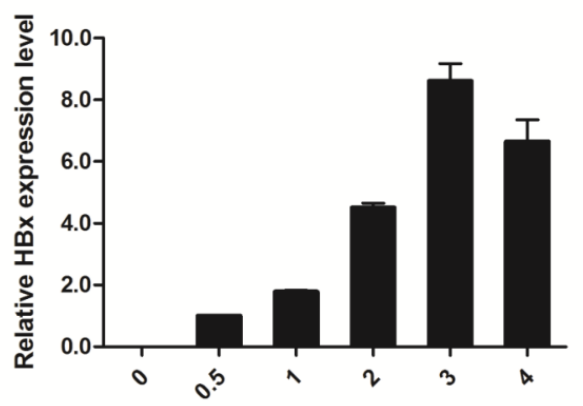

HBx transient transfection dose $(\mu \mathrm{g})$

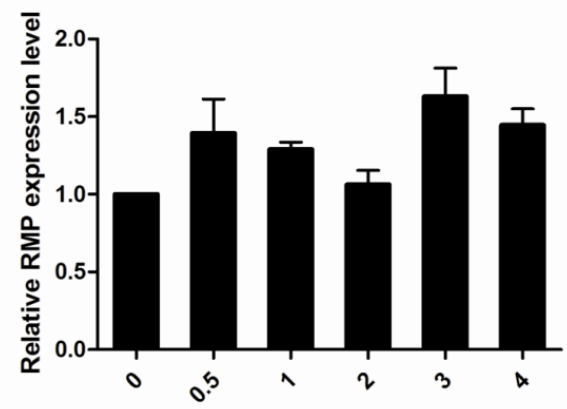

HBx transient transfection dose $(\mu \mathrm{g})$

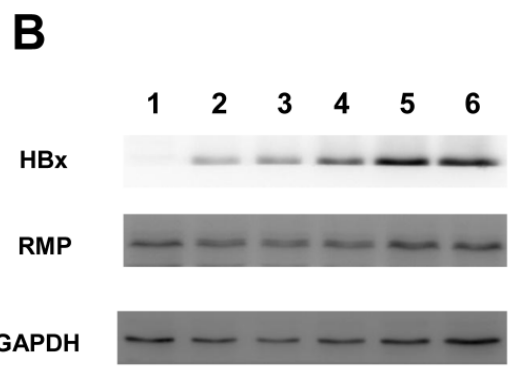

HBx (ug) $\quad \begin{array}{llllll}0 & 0.5 & 1 & 2 & 3 & 4\end{array}$
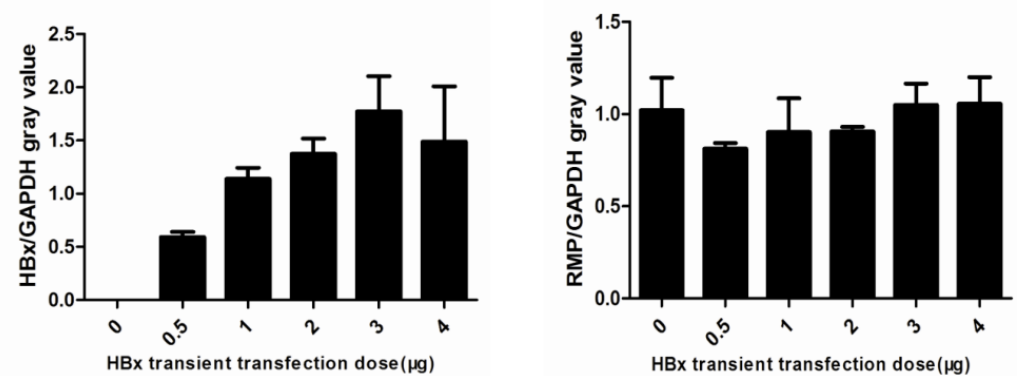

Fig. 5. HBx does not regulate the expression of RMP in HCC cells. (A). HepG2 cells were transfected with increasing amounts of HBx expression vector as indicated. qRT-PCR was performed for the quantative determination of the expression of HBx (left panel) and endogenous RMP (right panel). (B). HepG2 cells were transfected with increasing amounts of $\mathrm{HBx}$ expression vector as indicated. Cell lysates were extracted for the Western blot analysis (left panel) with antibodies against $\mathrm{HBx}$, RMP or GAPDH as indicated. The protein expression of $\mathrm{HBx}$ (middle panel) and RMP (right panel) was quantitated against GAPDH. Results are reported as means \pm S.D. of three independent experiments. 
A

Cy3-RMP
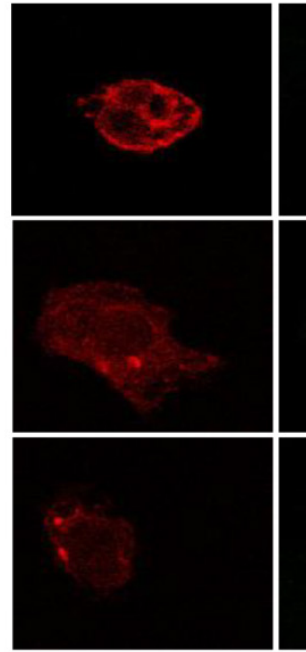

B

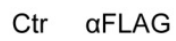

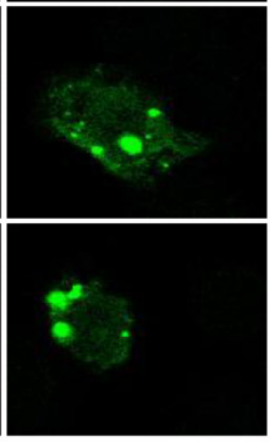

FITC-HBx

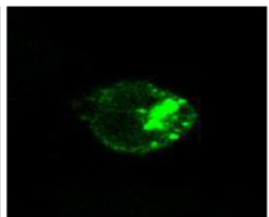

C
DAPI

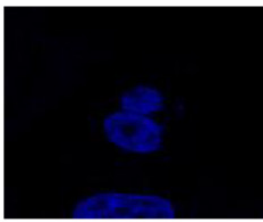

Merge

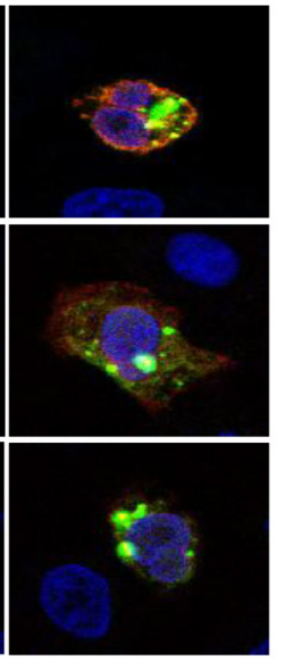

Ctr aHBx

$\mathrm{HBx}$

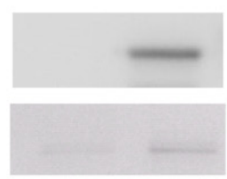

$\mathrm{HBx}$

RMP

Fig. 6. HBx interacts with RMP in HCC cells. (A). HepG2 cells were cotransfected with HBx and Flag-RMP expression vectors. Cells were fixed and immunostained with antibodies against RMP conjugated with $\mathrm{Cy} 3$ and $\mathrm{HBx}$ antibody conjugated with FITC. The stained cells were analyzed and photographed under a confocal laser scanning microscope. (B). Flag-RMP expression vectors were cotransfected into HepG2 cells stably expressing HBx. Cell lysates were extracted and immunoprecipitation was performed with antibody against Flag. The immunoprecipitates were fractionated in 12.5\% SDS-PAGE and subjected to Western blot analysis with antibodies against HBx and RMP as indicated. Preimmune IgG was applied as control of immunoprecipiation (Ctr). (C). Lysates were extracted from HepG2 cells stably expressing HBx. Immunoprecipitation was performed with antibody against HBx or with the preimmue IgG as a control (Ctr). The immunoprecipitates were fractionated in $12.5 \%$ SDS-PAGE and subjected to Western blot analysis with antibodies against $\mathrm{HBx}$ and RMP as indicated.

A

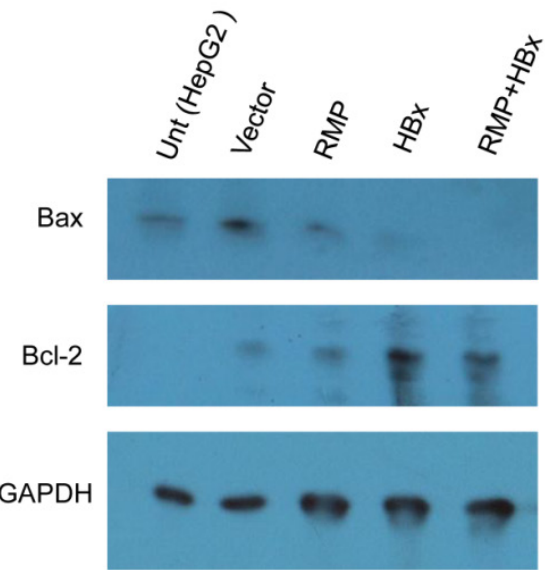

$\begin{array}{lllll}1 & 2 & 3 & 4 & 5\end{array}$
B

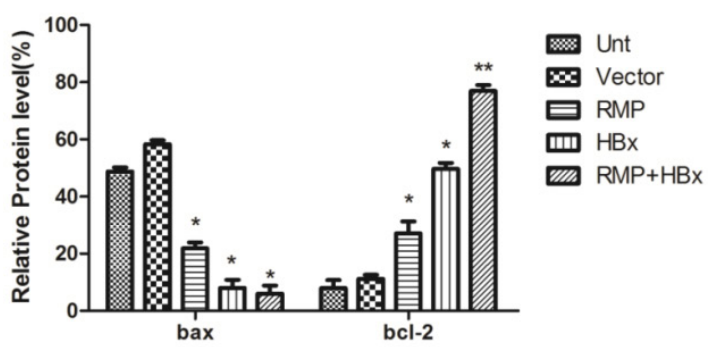

C

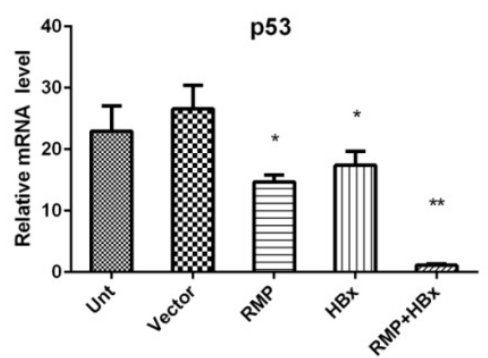

Fig. 7. HBx and RMP collaborately regulate apoptotic genes. (A). HepG2 cells (lane 1) were transfected with empty vector (lane 2), expression vectors for RMP (lane 3), HBx (lane 4), or both RMP and HBx (lane 5). Protein was extracted and Western blot analysis was carried out with antibodies against Bax, Bcl-2 and GAPDH which was applied for the normalization. The relative expression of Bax and Bcl-2 against GAPDH were graphically depicted in (B). (C). HepG2 cells were transfected with vectors as indicated. The mRNA was extracted and quantative RT-PCR was carried out for the determination of expression of $\mathrm{p} 53$. The expression of GAPDH was also determined for the normalization of expression of above factors. Student's t test, $*, p<0.05 ; * *, p<0.01$, relative to controls. 


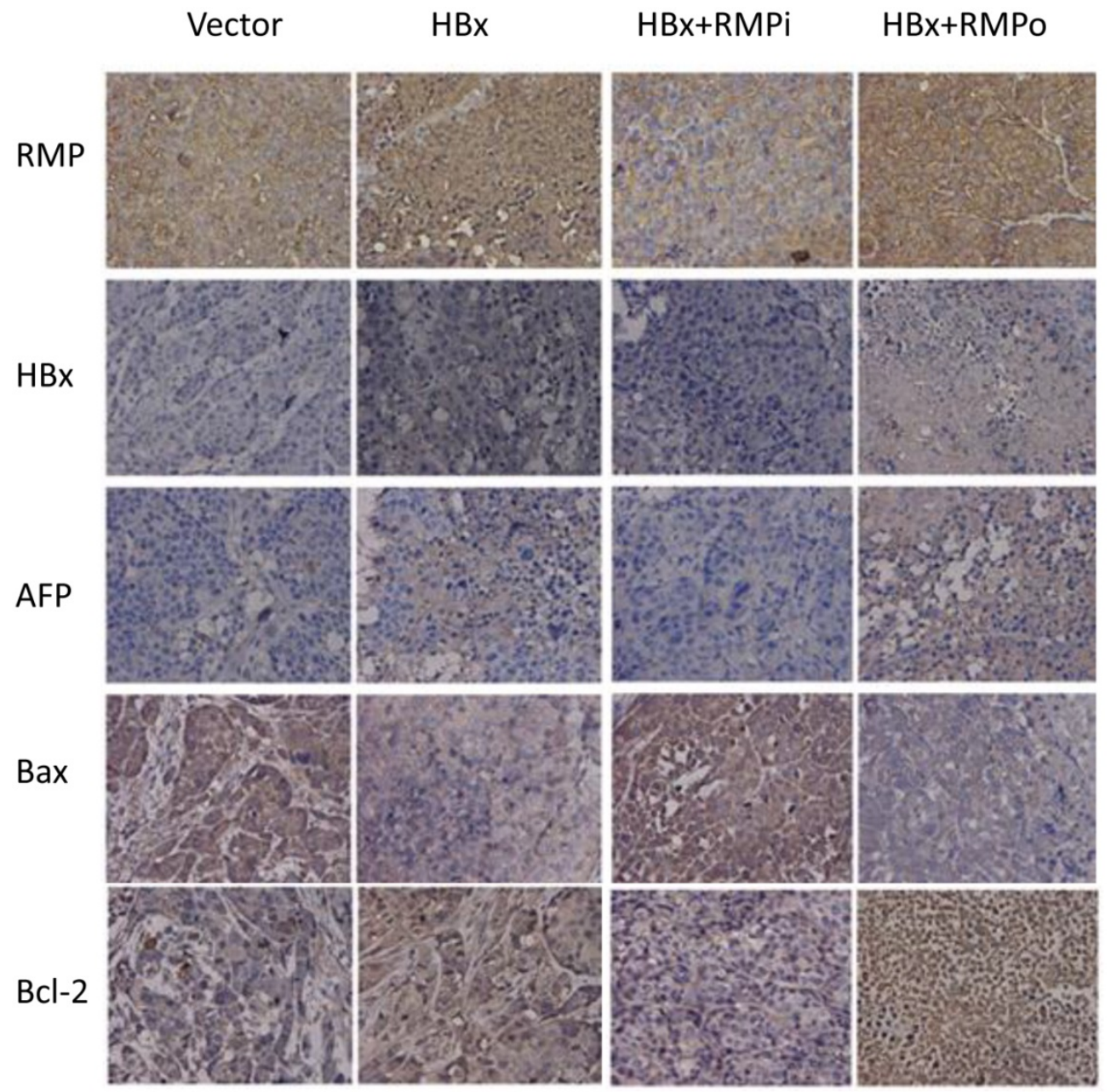

Fig. 8. Immunohistochemical analysis of expression of apoptotic factors in mouse xenograft tumors. HepG2 cells stably expressing HBx were inoculated subcutaneously into the dorsal flanks of nude mince. Two weeks later, the established tumors were subjected to intratumor injection with vectors as indicated on the top. Tumors were sectioned and immunohistochemical examination was carried out with antibodies as indicated to the left.

\section{Discussion}

RMP/URI has been shown to play multiple roles in transcription regulation $(8,18,19)$, nutrient-related signaling pathways (20) and maintenance of genomic stability (21). Recent studies identified that RMP/URI acts as an oncogene which is involved in the carcinogenesis of both HCC and ovarian cancer $(9,10)$.

RMP has multiple binding partners which might contribute to the complexity of RMP function. RMP binds with a multiprotein complex, including SKP2, PFD2, PFD4, RPB5 of the core subunit of RNAP II, the ATPases TIP48 and TIP49, which is within chromatin-remodeling complexes. RMP was found to be copurified with paf1 complex (16), which is involved in the histone methylation and cell cycle control. RMP interacts with DNA methyltransferase I (DNMT1)-associating protein (DMAP1), and is implicated in gene silencing (22). The association of yeast homologue of RMP (Bud27p) with the histone deme- thylase domain of Gis $1 p^{\prime}$ jmjC $(5,23)$, suggests that RMP might be involved in the epigenetic regulation.

HBx encoded by the HBV genome is crucial for the carcinogenesis of HCC. In the culture system, not only the transcription of viral genes, but also that of host genes, are activated by HBx. Similar to RMP, HBx is located mainly in cytosolic. Some cytosolic HBx are localized in mitochondria (1-3, 24-26), where RMP was located (11). Our results demonstrated the colocalization of RMP and HBx in the cytosol. In the cultured cells, HBx is localized in the outer mitochondrial membrane, which is consistent with the reported interaction of $\mathrm{HBx}$ with voltage-dependent anion channel (VDAC) spanning the outer mitochondrial membrane (2-4).

Although HBx does not bind directly to DNA, it may regulate gene expression via protein-protein interaction. Actually $\mathrm{HBx}$ has been shown to be involved in transcription (27), signaling pathways, protein degradation, cell-cycle control $(28,29)$, and carcinogenesis (30). Molecular evidence indicates that 
HBx is an oncoprotein. However, the detailed mechanism of HBx-mediated hepatocarcinogenesis with its partners remains unclear.

In this article, we report that HBx and RMP cooperatively stimulate the proliferation and suppress the apoptosis of HCC cells. Our studies show that HBx and RMP were colocalized in the HCC cells and could be co-immunoprecipitated. Consistent with the presence of physical interaction, functional collaboration was observed between HBx and RMP as well. The cell growth assay demonstrated that coexpression of both HBx and RMP resulted in a cooperative effect in the promotion of the HCC cell growth. A similar collaboration was observed on the apoptosis of HCC cells. Coexpression of HBx and RMP cooperatively suppressed the apoptosis of HCC. The collaboration between HBx and RMP was also observed in vivo by the nude mice experiments.

Apparently, apoptotic factors are the targets of both RMP and HBx. Our results demonstrated that RMP or HBx alone suppressed the expression of proapoptotic factor such as Bax, while at the same time enhancing the expression of antiapoptotic factors, such as Bcl-2. This is consistent with the reported result that Bcl-2 was elevated by the expression of HBx in a microarray (31). Coexpression of both RMP and $\mathrm{HBx}$ cooperatively regulates the expression of apoptotic factors.

Although much more clinical data is required to confirm the possible collaboration between $\mathrm{HBx}$ and RMP in the carcinogenesis of HCC, the immunohistochemical examination in HCC tissues demonstrated that enhanced expression of RMP and HBx was accompanied by reduced expression of proapoptotic factors, together with elevated expression of antiapoptotic factors.

There are several possible lines of mechanism for the cooperation between HBx and RMP, as common targets or pathways were identified to be shared by both HBx and RMP.

RMP has been found to function in the mTOR survival signaling. PP1 $\gamma$ is able to suppress the cell survival by inhibiting S6 kinase (S6K1) and BAD phosphorylation. RMP binds with PP1 $\gamma$ which is inhibited in the coupled state. S6K1 phosphorylates the $\mathrm{RMP}$, resulting in the release of PP1 $\gamma$ from the inactive state (11).

Interestingly, $\mathrm{HBx}$ has also been involved in the mTOR signaling. Overexpression of $\mathrm{HBx}$ in HCC cells upregulated mammalian target of rapamycin (mTOR) downstream effector S6 kinase (S6K1), and increased as well the cell proliferation and vascular endothelial growth factor (VEGF) production. HBx transgenic mouse model demonstrated that $\mathrm{HBx}$-modulated
mTOR/S6K1 signaling was associated with liver tumorigenesis (32).

Both HBx and RMP function in the DNA damage repair. HBx associates with the DNA damage-binding (DDB) protein, which binds to the breakends of damaged DNA (33). In C.elegans, RMP is required for the DNA stability and genome integrity. Depletion of RMP resulted in DNA damage and germline apoptosis in a p53-dependent manner (19).

Dr. Murakami group identified DNA methytransferase 1 (DNMT1)-associating protein (DMAP1) as one of the RMP-interacting partners. DMAP1 facilitated the nuclear localization of RMP and the corepressor activity of RMP in a dose-dependent manner (22). Interestingly, HBx has also been demonstrated to upregulate the expression of DNMT1 (34).

A similar cooperation was observed between two oncogenes of HBx and AIB1 (amplified in breast cancer 1) in the hepatocarcinogenesis of HCC (32). HBx was found to inhibit the ubiquitination and degradation of AIB1, therefore stabilize AIB1 protein and enhance its expression. Interestingly, recent studies show that $\mathrm{HBx}$ also upregulates oncogene expression of Rab18 and Lin28A/Lin28B and deregulates the proliferation of hepatoma cells $(13,14)$. However in our study, HBx did not affect the expression of endogenous RMP. Instead, the cooperation relies on the interaction between HBx and RMP, as verified by the inmmunoprecipitation and colocalizaion in HCC cells.

This finding may be of important significance, which may explain or partially explain the hepatocarcinogenesis by the infection of HBV. After HBV infection, $\mathrm{HBx}$ expresses and targets to the RPB5 of RNAP II, which in turn associated with the endogenous cellular oncoprotein RMP. The physical vicinity and interaction, between endogenous cellular oncoprotein RMP and $\mathrm{HBV}$ oncoprotein $\mathrm{HBx}$ leads to functional collaboration which inhibits the apoptosis of HCC cells, and contributes to the carcinogenesis of HCC. If this model is true, disruption of connections between $\mathrm{HBx}$ and RMP may be a potential way for the prevention and treatment of HCC.

\section{Acknowledgments}

This study was partly supported by Nature Science Foundation of China $(81172347,81272301)$, Special Research Found for the Doctoral Program of Higher Education (20123201110011), A project Funded by the Priority Academic Program Development of Jiangsu Higher Education Institutions and the Graduate Innovation Project of Jiangsu Province (CXZZ12-0834 and CXZZ13-0828). 


\section{Competing Interests}

The authors have declared that no competing interest exists.

\section{References}

1. Rawat S, Clippinger AJ, Bouchard MJ. Modulation of apoptotic signaling by the hepatitis B virus X protein. Viruses. 2012; 4: 2945-72. doi:10.3390/v4112945.

2. Clippinger AJ, Bouchard MJ. Hepatitis B virus HBx protein localizes to mitochondria in primary rat hepatocytes and modulates mitochondrial membrane potential. Journal of virology. 2008; 82: 6798-811. doi:10.1128/JVI.00154-08.

3. Rahmani Z, Huh KW, Lasher R, Siddiqui A. Hepatitis B virus X protein colocalizes to mitochondria with a human voltage-dependent anion channel, HVDAC3, and alters its transmembrane potential. Journal of virology. 2000; 74: $2840-6$.

4. Shoshan-Barmatz V, Israelson A, Brdiczka D, Sheu SS. The voltage-dependent anion channel (VDAC): function in intracellular signalling, cell life and cell death. Current pharmaceutical design. 2006; 12: 2249-70.

5. Miron-Garcia MC, Garrido-Godino AI, Garcia-Molinero V, Hernandez-Torres F, Rodriguez-Navarro S, Navarro F. The prefoldin bud27 mediates the assembly of the eukaryotic RNA polymerases in an rpb5-dependent manner. PLoS genetics. 2013; 9: e1003297. doi:10.1371/journal.pgen.1003297.

6. Woychik NA, Liao SM, Kolodziej PA, Young RA. Subunits shared by eukaryotic nuclear RNA polymerases. Genes \& development. 1990; 4: 313-23.

7. Cheong JH, Yi M, Lin Y, Murakami S. Human RPB5, a subunit shared by eukaryotic nuclear RNA polymerases, binds human hepatitis $\mathrm{B}$ virus $\mathrm{X}$ protein and may play a role in X transactivation. The EMBO journal. 1995; 14: 143-50.

8. Dorjsuren D, Lin Y, Wei W, Yamashita T, Nomura T, Hayashi N, et al. RMP, a novel RNA polymerase II subunit 5-interacting protein, counteracts transactivation by hepatitis B virus $\mathrm{X}$ protein. Molecular and cellular biology. 1998; 18: 7546-55.

9. Theurillat JP, Metzler SC, Henzi N, Djouder N, Helbling M, Zimmermann AK, et al. URI is an oncogene amplified in ovarian cancer cells and is required for their survival. Cancer cell. 2011; 19: 317-32. doi:10.1016/j.ccr.2011.01.019.

10. Yang H, Gu J, Zheng Q, Li M, Lian X, Miao J, et al. RPB5-mediating protein is required for the proliferation of hepatocellular carcinoma cells. The Journal of biological chemistry. 2011; 286: 11865-74. doi:10.1074/jbc.M110.136929.

11. Djouder N, Metzler SC, Schmidt A, Wirbelauer C, Gstaiger M, Aebersold R, et al. S6K1-mediated disassembly of mitochondrial URI/PP1gamma complexes activates a negative feedback program that counters S6K1 survival signaling. Molecular cell. 2007; 28: 28-40. doi:10.1016/j.molcel.2007.08.010.

12. Yang S, Wang H, Guo Y, Chen S, Zhang MY, Shen J, et al. RMP plays distinct roles in the proliferation of hepatocellular carcinoma cells and normal hepatic cells. International journal of biological sciences. 2013; 9: 637-48. doi:10.7150/ijbs.6439.

13. You $X$, Liu $F$, Zhang $T$, Li $Y$, Ye $L$, Zhang $X$. Hepatitis $B$ virus $X$ protein upregulates oncogene Rab18 to result in the dysregulation of lipogenesis and proliferation of hepatoma cells. Carcinogenesis. 2013; 34: 1644-52. doi:10.1093/carcin/bgt089.

14. You X, Liu F, Zhang T, Lv N, Liu Q, Shan C, et al. Hepatitis B virus X protein upregulates Lin28A/Lin28B through Sp-1/c-Myc to enhance the proliferation of hepatoma cells. Oncogene. 2013. doi:10.1038/onc.2012.618.

15. Gu J, Sun D, Zheng Q, Wang X, Yang H, Miao J, et al. Human Elongator complex is involved in cell cycle and suppresses cell growth in 293T human embryonic kidney cells. Acta biochimica et biophysica Sinica. 2009; 41: 831-8.

16. Yart A, Gstaiger M, Wirbelauer C, Pecnik M, Anastasiou D, Hess D, et al. The HRPT2 tumor suppressor gene product parafibromin associates with human PAF1 and RNA polymerase II. Molecular and cellular biology. 2005; 25: 5052-60. doi:10.1128/MCB.25.12.5052-5060.2005.

17. Liu $\mathrm{Y}$, Tong $\mathrm{Z}$, Li T, Chen $\mathrm{Q}$, Zhuo $\mathrm{L}$, Li W, et al. Hepatitis B virus $\mathrm{X}$ protein stabilizes amplified in breast cancer 1 protein and cooperates with it to promote human hepatocellular carcinoma cell invasiveness. Hepatology. 2012; 56: 1015-24. doi:10.1002/hep. 25751.

18. Wei W, Dorjsuren D, Lin Y, Qin W, Nomura T, Hayashi N, et al. Direct interaction between the subunit RAP30 of transcription factor IIF (TFIIF) and RNA polymerase subunit 5, which contributes to the association between TFIIF and RNA polymerase II. The Journal of biological chemistry. 2001; 276: 12266-73. doi:10.1074/jbc.M009634200.

19. Mita P, Savas JN, Djouder N, Yates JR, 3rd, Ha S, Ruoff R, et al. Regulation of androgen receptor-mediated transcription by RPB5 binding protein URI/RMP. Molecular and cellular biology. 2011; 31: 3639-52. doi:10.1128/MCB.05429-11.

20. Gstaiger M, Luke B, Hess D, Oakeley EJ, Wirbelauer C, Blondel M, et al. Control of nutrient-sensitive transcription programs by the unconventional prefoldin URI. Science. 2003; 302: 1208-12. doi:10.1126/science.1088401.

21. Parusel CT, Kritikou EA, Hengartner MO, Krek W, Gotta M. URI-1 is required for DNA stability in C. elegans. Development. 2006; 133: 621-9. doi:10.1242/dev.02235.

22. Delgermaa L, Hayashi N, Dorjsuren D, Nomura T, Thuy le TT, Murakami S. Subcellular localization of RPB5-mediating protein and its putative functional partner. Molecular and cellular biology. 2004; 24: 8556-66. doi:10.1128/MCB.24.19.8556-8566.2004.

23. Tronnersjo S, Hanefalk C, Balciunas D, Hu GZ, Nordberg N, Muren E, et al. The jmjN and jmjC domains of the yeast zinc finger protein Gis1 interact with 19 proteins involved in transcription, sumoylation and DNA repair. Molecular genetics and genomics : MGG. 2007; 277: 57-70. doi:10.1007/s00438-006-0171-3.

24. Kim S, Kim HY, Lee S, Kim SW, Sohn S, Kim K, et al. Hepatitis B virus x protein induces perinuclear mitochondrial clustering in microtubule- and Dynein-dependent manners. Journal of virology. 2007; 81: 1714-26. doi:10.1128/JVI.01863-06.

25. Huh KW, Siddiqui A. Characterization of the mitochondrial association of hepatitis B virus X protein, HBx. Mitochondrion. 2002; 1: 349-59.

26. Shirakata $Y$, Koike K. Hepatitis $B$ virus $X$ protein induces cell death by causing loss of mitochondrial membrane potential. The Journal of biological chemistry. 2003; 278: 22071-8. doi:10.1074/jbc.M301606200.

27. Lee SG, Rho HM. Transcriptional repression of the human p53 gene by hepatitis B viral X protein. Oncogene. 2000; 19: 468-71. doi:10.1038/sj.onc.1203312.

28. Jia L, Wang XW, Harris CC. Hepatitis B virus X protein inhibits nucleotide excision repair. International journal of cancer Journal international du cancer. 1999; 80: 875-9.

29. Bouchard MJ, Schneider RJ. The enigmatic $X$ gene of hepatitis $B$ virus. Journal of virology. 2004; 78: 12725-34. doi:10.1128/JVI.78.23.12725-12734.2004.

30. Cougot D, Neuveut C, Buendia MA. HBV induced carcinogenesis. Journal of clinical virology : the official publication of the Pan American Society for Clinical Virology. 2005; 34 Suppl 1: S75-8.

31. Zhang WY, Xu FQ, Shan CL, Xiang R, Ye LH, Zhang XD. Gene expression profiles of human liver cells mediated by hepatitis $\mathrm{B}$ virus $\mathrm{X}$ protein. Acta pharmacologica Sinica. 2009; 30: 424-34. doi:10.1038/aps.2009.22.

32. Yen CJ, Lin YJ, Yen CS, Tsai HW, Tsai TF, Chang KY, et al. Hepatitis B virus X protein upregulates mTOR signaling through IKKbeta to increase cell proliferation and VEGF production in hepatocellular carcinoma. PloS one. 2012; 7: e41931. doi:10.1371/journal.pone.0041931.

33. Lee TH, Elledge SJ, Butel JS. Hepatitis B virus $X$ protein interacts with a probable cellular DNA repair protein. Journal of virology. 1995; 69: 1107-14.

34. Zheng DL, Zhang L, Cheng N, Xu X, Deng Q, Teng XM, et al. Epigenetic modification induced by hepatitis $B$ virus $X$ protein via interaction with de novo DNA methyltransferase DNMT3A. Journal of hepatology. 2009; 50: 377-87. doi:10.1016/j.jhep.2008.10.019. 